
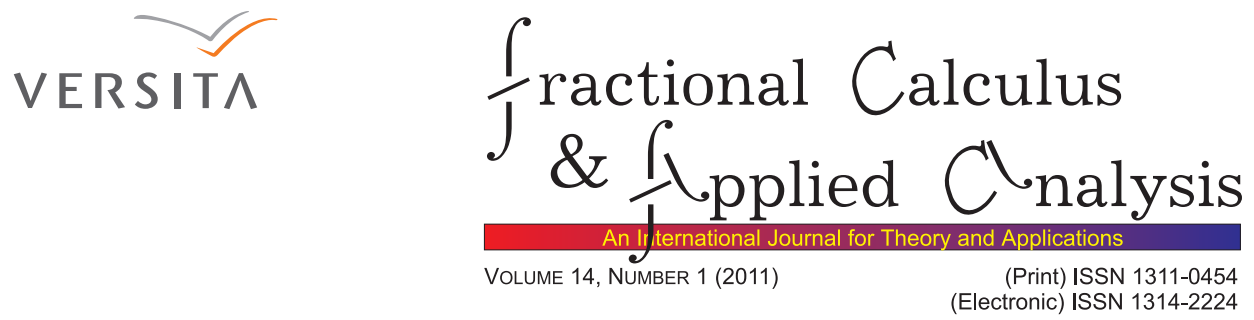

RESEARCH PAPER

\title{
ON A 3D-HYPERSINGULAR EQUATION \\ OF A PROBLEM FOR A CRACK
}

\section{Stefan Samko}

\begin{abstract}
Dedicated to Professor R. Gorenflo on the occasion of his 80th birthday

We show that a certain axisymmetric hypersingular integral equation arising in problems of cracks in the elasticity theory may be explicitly solved in the case where the crack occupies a plane circle. We give three different forms of the resolving formula. Two of them involve regular kernels, while the third one involves a singular kernel, but requires less regularity assumptions on the the right-hand side of the equation.
\end{abstract}

MSC 2010: 45DB05, 45E05, 78A45

Key Words and Phrases: fractional operator, hypersingular integrals, diffraction, cracks, potential kernel, singular operator

\section{Introduction}

A static $3 d$ problem for a crack in elastic media ([10]) in a scalar approximation is known to be described by the following hypersingular integral equation of the potential theory

$$
\int_{S} \frac{\partial^{2}}{\partial n_{\mathbf{x}} \partial n_{\mathbf{y}}}\left(\frac{1}{|\mathbf{y}-\mathbf{x}|}\right) u(\mathbf{y}) d S_{\mathbf{y}}=f(\mathbf{x}), \quad \mathbf{x} \in S,
$$

where $\mathbf{n}_{\mathbf{x}}, \mathbf{n}_{\mathbf{y}}$ stand for the normal vectors at the points $\mathbf{x}, \mathbf{y}$ of the surface $S$, $f(\mathbf{x}) \in C^{\infty}(\ell)$ and the crack surface $S$ is usually assumed to be smooth.

(c) 2011 Diogenes Co., Sofia

pp. 19-30, DOI: 10.2478/s13540-011-0003-y 
We do not dwell on details related to the proper interpretation of the divergent hypersingular integral in (2.1), this follows the standard ideas based either on the Hadamard finite part or treating the hypersingular integral as a result of application of a differential operator to the corresponding integral operator, both ways coinciding in the case under the consideration, we refer to [8] for details.

In applications there are known numerical methods applied directly to equations of such a type, see for instance [2]. In this note we consider a special case where the crack is plane and occupies a circular region $B=$ $\left\{\mathbf{x} \in \mathbb{R}^{2}:|\mathbf{x}|<R\right\}$. We show that in this case it is possible to obtain the explicit solution in the closed form. For simplicity we consider - in this paper - the axisymmetric case.

\section{Some general facts}

\subsection{Reduction to the inversion of the potential operator}

We start with the study of a more general hypersingular integral equation

$$
\iint_{\Omega} \frac{u(\mathbf{y}) d y}{|\mathbf{x}-\mathbf{y}|^{2+\alpha}}=f(\mathbf{x}), \quad x \in \Omega
$$

where $\Omega$ is a plane region and we deal with the case $0<\alpha<2$, but the main our interest will be related to the case $\alpha=1$ corresponding to the crack problem, and the case $\Omega=B(0, R)$. We suppose that $f(\mathbf{x}) \in C^{\infty}(\Omega)$ and look for bounded solutions $u$.

The strong singularity above may be treated as a result of the application of the Laplace operator to a weaker singularity. Let

$$
\Delta_{x}=\frac{\partial^{2}}{\partial^{2} x_{1}}+\frac{\partial^{2}}{\partial^{2} x_{2}} \quad \text { and } \quad r=|\mathbf{x}-\mathbf{y}| .
$$

For functions $\varphi=\varphi(r)$ depending on the distance, the Laplacian is reduced to

which in particular gives

$$
\Delta_{x} \varphi=\varphi^{\prime \prime}(r)+\frac{1}{r} \varphi^{\prime}(r)
$$

$$
\Delta_{x}\left(r^{\beta}\right)=\beta^{2} r^{\beta-2}
$$

where $\beta \in \mathbb{R}^{1}$. Therefore, we will interpret equation (2.1) as

$$
\Delta_{x}\left(\iint_{\Omega} \frac{u(\mathbf{y}) d y}{|\mathbf{x}-\mathbf{y}|^{\alpha}}\right)=f_{1}(\mathbf{x}), \quad \mathbf{x} \in \Omega
$$

where $f_{1}(\mathbf{x})=\alpha^{2} f(\mathbf{x})$ and the integral already exists in the usual sense.

As is known, a particular solution of the Laplace equation

$$
\Delta \varphi=f_{1}
$$


in the planar case is given by the Newtonian potential

$$
\varphi(\mathbf{x})=-\frac{1}{2 \pi} \iint_{\Omega} f_{1}(y) \ln \frac{1}{|\mathbf{x}-y|} d y=: g(\mathbf{x}) .
$$

Therefore, equation (2.2) is reduced to

$$
\iint_{\Omega} \frac{u(\mathbf{y}) d y}{|\mathbf{x}-\mathbf{y}|^{\alpha}}=g(\mathbf{x})+h(\mathbf{x})
$$

where $h(\mathbf{x})$ is an arbitrary function harmonic in $\Omega$.

Let us temporarily forget about this problem of the unique determination of $h(\mathbf{x})$ and suppose that we know the right hand side

$$
f_{2}(\mathbf{x})=g(\mathbf{x})+h(\mathbf{x})
$$

of equation (2.3) is known. We have arrived at the problem of the inversion of the equation

$$
\iint_{\Omega} \frac{u(\mathbf{y}) d y}{|\mathbf{x}-\mathbf{y}|^{\alpha}}=f_{2}(\mathbf{x})
$$

In general, there are not known direct approaches to solve such equations of the first kind for arbitrary domains $\Omega$. For an arbitrary domains one could use the approach of the paper 3 which only allows to reduce such an equation to a singular type equation of the 2 nd type. This approach is based on the usage of the multidimensional Marchaud type formula

$$
\mathbb{D}_{\Omega}^{\alpha} f(x)=c(\alpha)\left[a_{\Omega}(x) f(x)+\int_{\Omega} \frac{f(x)-f(y)}{|x-y|^{n+\alpha}} d y\right], \quad x \in \Omega,
$$

where $0<\alpha<1$,

$$
a_{\Omega}(x)=\int_{\mathbb{R}^{n} \backslash \Omega} \frac{d y}{|x-y|^{n+\alpha}} \quad \text { and } \quad c(\alpha)=\frac{2^{\alpha} \Gamma\left(1+\frac{\alpha}{2}\right) \Gamma\left(\frac{n+\alpha}{2}\right) \sin \frac{\alpha \pi}{2}}{\pi^{1+\frac{n}{2}}} .
$$

for domains in $\mathbb{R}^{n}$ adjusted for the Riesz fractional derivatives, see about Riesz fractional derivatives $\equiv$ hypersingular integrals in [9], Section 26. The equation

$$
\mathbb{D}_{\Omega}^{\alpha}\left(\iint_{\Omega} \frac{u(\mathbf{y}) d y}{|\mathbf{x}-\mathbf{y}|^{\alpha}}\right)=\mathbb{D}_{\Omega}^{\alpha} f_{2}(x)
$$

may be realized as an integral equation of the 2nd kind with a singular kernel. However, the realization of this method and the separation of the singular part of the kernel heavily depend on the properties of the boundary. 
REMARK 2.1. Note that solutions of equations of the type (2.4) are usually unbounded near the boundary $\partial \Omega$ of the domain $\Omega$ and no smoothness condition on the right-hand side $f_{2}(\mathbf{x})$ guarantees the boundedness of the solution. It is expected that some orthogonality condition for $f_{2}(\mathbf{x})$ should hold in order to have a bounded solution, see in particular Remark 3.1 .

\subsection{The case $n=2$ and $\Omega=B(0, R)$}

We now return to the case $\Omega=B=B(0, R)$ when (2.3) is

$$
\iint_{|\mathbf{y}|<R} \frac{u(\mathbf{y}) d y}{|\mathbf{x}-\mathbf{y}|^{\alpha}}=g(\mathbf{x})+h(\mathbf{x}),
$$

where $h(\mathbf{x})$ is an arbitrary harmonic function in $B$ (integrable on $B$ ) which should be determined basing on some additional conditions, and $g(\mathbf{x})$ is given by the logarithmic potential

$$
g(\mathbf{x})=\frac{\alpha^{2}}{2 \pi} \int_{B} f(\mathbf{y}) \ln |\mathbf{x}-\mathbf{y}| d y .
$$

In the case of the circle, the method of solution of equation (2.6) is known in the closed form, see [6] or [7], Section 27. It is based on the fact that the potential operator over a ball may be factorized. Namely, it may be represented as a composition of the so called unilateral ball potentials which may be inverted by the analogy with the Riemann-Liouville fractional operators.

We will not consider this general approach in this paper. Now we intend to treat the angular-invariant (axisymmetric) case, that is, we suppose that the right-hand side $f=f(|\mathbf{x}|)$ is radial and correspondingly we look for radial type solutions $u(|\mathbf{y}|)$.

\section{An explicit formula for axisymmetric (radial) solutions}

\subsection{The 1st formula}

Let $f=f(|\mathbf{x}|)$ be radial and we look for radial solutions $u(|\mathbf{y}|)$. It is easy to see that $g(\mathbf{x})$ is also radial. Similarly, the left-hand side of (2.6) depends only on $|\mathbf{x}|$. Therefore, the function $h(\mathbf{x})$ in $(2.6)$ is radial. Since

$$
\Delta h(r)=h^{\prime \prime}(r)+\frac{h^{\prime}(r)}{r}, \quad r=|\mathbf{x}|,
$$


radial harmonic functions may be only of the form $h(r)=c_{1} \ln r+c_{2}$. Since we re looking for bounded solutions $u$, we may take only $h(r)=$ const. Consequently, equation (2.6) takes the form

$$
\iint_{|\mathbf{y}|<R} \frac{u(|\mathbf{y}|) d y}{|\mathbf{x}-\mathbf{y}|^{\alpha}}=g|\mathbf{x}|+C
$$

the constant $C$ will be determined later. It remains to solve equation (3.1), which is the problem of inversion of potential type operators.

From now on, we take $\alpha=1$.

LEMma 3.1. In the case $f=f(|\mathbf{x}|)$ is radial, the function $g=g(|\mathbf{x}|)$ defined in (2.7), is given by the formula

$$
g(r)=\ln r \int_{0}^{r} t f(t) d t+\int_{r}^{R} t f(t) \ln t d t
$$

so that

$$
g^{\prime}(r)=\frac{1}{r} \int_{0}^{r} t f(t) d t
$$

P r o o f. We pass to polar coordinates and obtain

$$
g(r)=\frac{1}{4 \pi} \int_{0}^{R} \varrho f(\varrho) d \varrho \int_{0}^{2 \pi} \ln \left|r^{2}-2 r \varrho \cos \theta+\varrho^{2}\right| d \theta .
$$

Making use of formula 4.224.14 from [1], we arrive at (3.2).

Theorem 3.1. Let $f \in C([0, R])$. All the integrable solutions of the equation

$$
\iint_{|\mathbf{y}|<R} \frac{u(|\mathbf{y}|) d y}{|\mathbf{x}-\mathbf{y}|^{3}}=g(|\mathbf{x}|)+C,
$$

where $C$ is a constant, are given by the formula

$$
u(r)=-G(r)+\frac{C}{\pi^{2} \sqrt{R^{2}-r^{2}}},
$$

where

$$
G(r):=\frac{1}{\pi^{2} r} \frac{d}{d r} \int_{r}^{R} \frac{d s}{\sqrt{s^{2}-r^{2}}} \int_{0}^{s} \frac{t g_{0}^{\prime}(t) d t}{\sqrt{s^{2}-t^{2}}}, \quad g_{0}(t):=\operatorname{tg}(t) .
$$


P r o o f. In case of balls (or circles in our case) and radial functions, the inversion formula for potentials is rather constructive. It is known for an arbitrary dimension $d \geq 2$ and the general kernel $\frac{1}{|\mathbf{x}-\mathbf{y}|^{d+\alpha}}$ and runs as follows:

$$
u(|\mathbf{x}|)=\frac{\Gamma\left(\frac{d-\alpha}{2}\right)}{\pi^{\frac{d}{2}} \Gamma\left(\frac{\alpha}{2}\right)}\left(D_{R^{2}-}^{\frac{\alpha}{2}} s^{1-\frac{d-\alpha}{2}} D_{0+}^{\frac{\alpha}{2}} t^{\frac{d}{2}-1}[g(\sqrt{t})+C]\right)\left(|\mathbf{x}|^{2}\right),
$$

see [5], where

$$
D_{0+}^{\frac{\alpha}{2}} \varphi(r)=\frac{1}{\Gamma\left(\frac{\alpha}{2}\right)} \frac{d}{d r} \int_{0}^{r} \frac{\varphi(\rho) d \rho}{(r-\rho)^{\frac{\alpha}{2}}}, \quad D_{R^{2}-}^{\frac{\alpha}{2}} \varphi(r)=-\frac{1}{\Gamma\left(\frac{\alpha}{2}\right)} \frac{d}{d r} \int_{r}^{R^{2}} \frac{\varphi(\rho) d \rho}{(\rho-r)^{\frac{\alpha}{2}}}
$$

are the Riemann-Liouville fractional derivatives of order $\frac{\alpha}{2}(0<\alpha<2)$; this inversion formula in the case $d=2$ was obtained by Rostovtsev, see [4].

In the case $d=2$ and $\alpha=1$, the inversion formula (3.6) takes the form

$$
u(r)=-\frac{1}{\pi^{2} r} \frac{d}{d r} \int_{r}^{R} \frac{s}{\sqrt{s^{2}-r^{2}}} \frac{d}{d s}\left(\int_{0}^{s} \frac{t[g(t)+C]}{\sqrt{s^{2}-t^{2}}} d t\right) d s, \quad r=|\mathbf{x}| .
$$

After easy transformations we arrive at (3.4)-(3.5).

Note that $f \in C \Longrightarrow g \in C^{2}$.

REMARK 3.1. As seen from (3.4), the obtained solutions are in general unbounded at the boundary $|\mathbf{x}|=R$, independently of the smoothness of the right-hand side $f(x)$ of the initial equation. Clearly an appropriate choice of the constant $C$ in (3.4) will provide a bounded solution.

\subsection{The 2nd formula}

Formula (3.4) for the solution involves repeated integration. It is possible to represent this solution in the form of a single integration in terms of the second derivative of $g(t)$. Namely, the following lemma holds, where we use the notation

$$
(\mathbb{D} g)(t)=\frac{d}{d t}[t g(t)], \quad \text { so that } \quad t\left(\mathbb{D}^{2} g\right)(t)=t g_{0}^{\prime}(t)+t^{2} g_{0}^{\prime \prime}(t)
$$

and

$$
F(\varphi, k):=\int_{0}^{\varphi} \frac{d \alpha}{\sqrt{1-k^{2} \sin ^{2} \alpha}}, \quad k^{2}<1
$$

for the elliptic integral. 
Lemma 3.2. Let $g \in C^{2}(0, R)$. Then the bounded solution of equation (3.3) may be represented as

$$
u(r)=\frac{1}{\pi^{2} r^{2}}\left(-\int_{0}^{R} \mathcal{K}(r, t) t\left(\mathbb{D}^{2} g\right)(t) d t+\frac{\sqrt{R^{2}-r^{2}}}{R} \int_{0}^{R} \frac{t g_{0}^{\prime}(t) d t}{\sqrt{R^{2}-t^{2}}}\right),
$$

where

$$
\mathcal{K}(r, t)= \begin{cases}\frac{1}{r} F\left(\arcsin \sqrt{\frac{R^{2}-r^{2}}{R^{2}-t^{2}}}, \frac{t}{r}\right), \quad t<r \\ \frac{1}{t} F\left(\arcsin \sqrt{\frac{R^{2}-t^{2}}{R^{2}-r^{2}}}, \frac{r}{t}\right), \quad t>r\end{cases}
$$

P r o o f. We need to simplify the function $G(r)$ from (3.4) and provide its expression in terms of a single integral of derivatives of $g(t)$. By the change of variables $t=s \tau$ and $s=r \sigma$, we have

$$
G(r)=\frac{1}{\pi^{2} r} \frac{d}{d r}\left(r \int_{1}^{\frac{R}{r}} \frac{\sigma d \sigma}{\sqrt{\sigma^{2}-1}} \int_{0}^{1} \frac{g_{0}^{\prime}(r \tau \sigma)}{\sqrt{1-\tau^{2}}} \tau d \tau\right) .
$$

After differentiation in $r$ we obtain

$$
\begin{gathered}
G(r)=\frac{1}{\pi^{2} r}\left(\int_{1}^{\frac{R}{r}} \frac{\sigma d \sigma}{\sqrt{\sigma^{2}-1}} \int_{0}^{1} \frac{g_{0}^{\prime}(r \tau \sigma)}{\sqrt{1-\tau^{2}}} \tau d \tau-\frac{R^{2}}{r \sqrt{R^{2}-r^{2}}} \int_{0}^{1} \frac{g_{0}^{\prime}(R \tau) \tau d \tau}{\sqrt{1-\tau^{2}}}\right. \\
\left.+r \int_{1}^{\frac{R}{r}} \frac{\sigma^{2} d \sigma}{\sqrt{\sigma^{2}-1}} \int_{0}^{1} \frac{\tau^{2} g_{0}^{\prime \prime}(r \sigma \tau) d \tau}{\sqrt{1-\tau^{2}}}\right)
\end{gathered}
$$

Then after the corresponding reverse changes of variables we get

$$
\begin{gathered}
G(r)=\frac{1}{\pi^{2} r}\left(\frac{1}{r} \int_{r}^{R} \frac{d s}{\sqrt{s^{2}-r^{2}}} \int_{0}^{s} \frac{t g_{0}^{\prime}(t) d t}{\sqrt{s^{2}-t^{2}}}-\frac{R}{r \sqrt{R^{2}-r^{2}}} \int_{0}^{R} \frac{t g_{0}^{\prime}(t) d t}{\sqrt{R^{2}-t^{2}}}\right. \\
\left.+\frac{1}{r} \int_{r}^{R} \frac{d s}{\sqrt{s^{2}-r^{2}}} \int_{0}^{s} \frac{t^{2} g_{0}^{\prime \prime}(t)}{\sqrt{s^{2}-t^{2}}} d t\right) .
\end{gathered}
$$

The first and the third integral may be united and we get

$$
G(r)=\frac{1}{\pi^{2} r^{2}}\left(\int_{r}^{R} \frac{d s}{\sqrt{s^{2}-r^{2}}} \int_{0}^{s} \frac{t\left(\mathbb{D}^{2} g\right)(t) d t}{\sqrt{s^{2}-t^{2}}}-\frac{R}{\sqrt{R^{2}-r^{2}}} \int_{0}^{R} \frac{t g_{0}^{\prime}(t) d t}{\sqrt{R^{2}-t^{2}}}\right),
$$


Now we have to transform the double integral

$$
\begin{gathered}
J(r):=\int_{r}^{R} \frac{d s}{\sqrt{s^{2}-r^{2}}} \int_{0}^{s} \frac{t\left(\mathbb{D}^{2} g\right)(t) d t}{\sqrt{s^{2}-t^{2}}} \\
=\int_{0}^{r} t\left(\mathbb{D}^{2} g\right)(t) d t \int_{r}^{R} \frac{d s}{\sqrt{s^{2}-r^{2}} \sqrt{s^{2}-t^{2}}}+\int_{r}^{R} t\left(\mathbb{D}^{2} g\right)(t) d t \int_{t}^{R} \frac{d s}{\sqrt{s^{2}-r^{2}} \sqrt{s^{2}-t^{2}}} \\
=\int_{0}^{R} t\left(\mathbb{D}^{2} g\right)(t) d t \int_{\max (r, t)}^{R} \frac{d s}{\sqrt{s^{2}-r^{2}} \sqrt{s^{2}-t^{2}}} .
\end{gathered}
$$

The inner integral

$$
\mathcal{K}(r, t):=\int_{\max (r, t)}^{R} \frac{d s}{\sqrt{s^{2}-r^{2}} \sqrt{s^{2}-t^{2}}}
$$

has the form

$$
\mathcal{K}(r, t)=\frac{1}{r} \begin{cases}\int_{1}^{\frac{R}{r}} \frac{d s}{\sqrt{s^{2}-1} \sqrt{s^{2}-\frac{t^{2}}{r^{2}}}}, & t<r, \\ \int_{1}^{\frac{R}{t}} \frac{d s}{\sqrt{s^{2}-1} \sqrt{\frac{t^{2}}{r^{2}} s^{2}-1}}, & t>r\end{cases}
$$

By formula 3.152.11 from [1], the kernel $\mathcal{K}(r, t)$ may be expressed in terms of the elliptic integral $F(\varphi, k)$ :

$\mathcal{K}(r, t)=\frac{1}{a} F\left(\mu, \frac{b}{a}\right)$ and $\mu=\arcsin \sqrt{\frac{R^{2}-a^{2}}{R^{2}-b^{2}}}, a=\max (t, r), b=\min (t, r)$,

so that we arrive at (3.8). Note that in particular,

$$
\mathcal{K}(0, t)=\frac{1}{t} \arccos \frac{t}{R} .
$$

From (3.4) we obtain

$$
u(r)=-\frac{1}{\pi^{2} r^{2}} \int_{0}^{R} \mathcal{K}(r, t) t\left(\mathbb{D}^{2} g\right)(t) d t+\frac{1}{\pi^{2} \sqrt{R^{2}-r^{2}}}\left[C+\frac{R}{r^{2}} \int_{0}^{R} \frac{t g_{0}^{\prime}(t) d t}{\sqrt{R^{2}-t^{2}}}\right] .
$$

The first term in the above formula has no singularity when $r \rightarrow R$, so that we arrive at the following choice of the constant $C$ to get a bounded 
solution:

$$
C=-\frac{1}{R} \int_{0}^{R} \frac{t g_{0}^{\prime}(t) d t}{\sqrt{R^{2}-t^{2}}}
$$

which leads us to (3.7).

REMARK 3.2. The solution $u(r)$ given by formula (3.7) has no singularity at the point $r=0$.

P r o o f. First we show that the expression in the parenthesis in (3.7) is equal to zero at the point $r=0$. To this end, we have to check that

$$
\int_{0}^{R}\left(\mathbb{D}^{2} g\right)(t) \arccos \frac{t}{R} d t=\int_{0}^{R} \frac{t g_{0}^{\prime}(t) d t}{\sqrt{R^{2}-t^{2}}}
$$

according to (3.12). The latter is easily checked by integration by parts.

It remains to observe that the derivative in $r$ of the expression in the parenthesis in (3.7) is equal to zero at the point $r=0$. This is obvious for the last term in that expression, and follows from the fact that $\mathcal{K}_{r}^{\prime}(0, t) \equiv 0$ for the first term.

\subsection{The 3rd formula}

It is natural to try to transform the 2nd formula for the solution, by diminishing the order of the used derivatives of $g(t)$; however this will lead to the appearance of the singular kernel. Indeed from the expression of the kernel $\mathcal{K}(r, t)$ via the elliptic integral, one may conclude that it has a logarithmic singularity of the type $\ln |t-r|$ as $t \rightarrow r$. Hence we expect that the derivative $\frac{\partial K}{\partial t}$ must be a singular kernel.

In the following lemma, besides the elliptic integral $F(\varphi, k)$ of the first kind, which appeared in the expression for the kernel $\mathcal{K}(r, t)$, we will also the elliptic integral

$$
E=E(\varphi, k):=\int_{0}^{\varphi} \sqrt{1-k^{2} \sin ^{2} \alpha} d \alpha
$$

of the second kind. Recall that

$$
\varphi=\left\{\begin{array}{ll}
\arcsin \sqrt{\frac{R^{2}-r^{2}}{R^{2}-t^{2}}}, & t<r \\
\arcsin \sqrt{\frac{R^{2}-t^{2}}{R^{2}-r^{2}},} & t>r,
\end{array} \quad \text { and } \quad k= \begin{cases}\frac{t}{r}, & t<r \\
\frac{r}{t}, & t>r\end{cases}\right.
$$


LEMma 3.3. The solution given in (3.7) may be represented in the form

$$
u(r)=\frac{1}{\pi^{2}} \int_{0}^{R} \mathcal{L}(r, t)[\operatorname{tg}(t)]^{\prime} d t
$$

where

$$
\mathcal{L}(r, t)=\frac{t}{r^{2}}\left[\frac{1}{R} \sqrt{\frac{R^{2}-r^{2}}{R^{2}-t^{2}}}+\frac{\partial}{\partial t}[t \mathcal{K}(r, t)]\right] .
$$

This kernel has a singular component:

$$
\mathcal{L}(r, t)=\frac{1}{r^{2}}\left[\frac{t \max \{t, r\}}{r^{2}-t^{2}} E+M(r, t)\right],
$$

where the "regular" component has the form

$$
M(r, t)= \begin{cases}\frac{t}{R} \sqrt{\frac{R^{2}-r^{2}}{R^{2}-t^{2}}}, & t<r, \\ F-\frac{t r^{2}}{R \sqrt{R^{2}-r^{2}} \sqrt{R^{2}-t^{2}}}, & t>r\end{cases}
$$

and $E=E(\varphi, k)$ and $F=F(\varphi, k)$ are elliptic integrals with arguments $\varphi$ and $k$ given in (3.13).

P r o o f. We integrate by parts in (3.7), taking into account that

$$
\left.\left.t \mathcal{K}(r, t)\right|_{t=0} \equiv t \mathcal{K}(r, t)\right|_{t=R}=0,
$$

which yields (3.14) after easy transformations.

We have to single out the singular component of the expression on the right-hand side of (3.15).

The case $t<r$. Recall that

$$
\mathcal{K}(r, t)= \begin{cases}\frac{1}{r} F(\varphi, k), & t<r \\ \frac{1}{t} F(\varphi, k), & t>r\end{cases}
$$

by (3.8). We have

$$
\frac{\partial \mathcal{K}(r, t)}{\partial t}=\frac{1}{r}\left(\frac{\partial F}{\partial \varphi} \frac{\partial \varphi}{\partial t}+\frac{\partial F}{\partial k} \frac{\partial k}{\partial t}\right) .
$$

Via direct calculations, we obtain

$$
\frac{\partial F}{\partial \varphi}=\frac{1}{\sqrt{1-k^{2} \sin ^{2} \varphi}}=\frac{r}{R} \sqrt{\frac{R^{2}-t^{2}}{r^{2}-t^{2}}},
$$

and

$$
\frac{\partial \varphi}{\partial t}=\frac{t}{R^{2}-t^{2}} \sqrt{\frac{R^{2}-r^{2}}{r^{2}-t^{2}}}, \quad \frac{\partial k}{\partial t}=\frac{1}{r} .
$$


By formula 8.123.1 from [1] we also have

$$
\frac{\partial F}{\partial k}=\frac{1}{k}\left(\frac{E}{1-k^{2}}-F\right)-\frac{k}{1-k^{2}} \frac{\sin \varphi \cos \varphi}{\sqrt{1-k^{2} \sin ^{2} \varphi}} .
$$

Consequently,

$$
\frac{\partial F}{\partial k}=\frac{r}{t}\left(\frac{r^{2} E}{r^{2}-t^{2}}-F\right)-\frac{t r^{2}}{R\left(r^{2}-t^{2}\right)} \sqrt{\frac{R^{2}-r^{2}}{R^{2}-t^{2}}} .
$$

We substitute this into (3.17) and obtain

$$
\frac{\partial \mathcal{K}(r, t)}{\partial t}=\frac{1}{r t}\left(\frac{r^{2}}{r^{2}-t^{2}} E-F\right) \text {. }
$$

Then

$$
\frac{\partial[t \mathcal{K}(r, t)]}{\partial t}=\frac{r E}{r^{2}-t^{2}} .
$$

The case $t>r$. We have

$$
\frac{\partial[t \mathcal{K}(r, t)]}{\partial t}=\frac{\partial[F(\varphi, k)]}{\partial t}=\frac{\partial F}{\partial \varphi} \frac{\partial \varphi}{\partial t}+\frac{\partial F}{\partial k} \frac{\partial k}{\partial t} .
$$

and in this case

$$
\frac{\partial F}{\partial \varphi}=\frac{1}{\sqrt{1-k^{2} \sin ^{2} \varphi}}=\frac{t}{R} \sqrt{\frac{R^{2}-r^{2}}{t^{2}-r^{2}}}
$$

and

$$
\frac{\partial \varphi}{\partial t}=\frac{-t}{\sqrt{R^{2}-t^{2}} \sqrt{t^{2}-r^{2}}}, \quad \frac{\partial k}{\partial t}=-\frac{r}{t^{2}} .
$$

By formula (3.18) we obtain

$$
\frac{\partial F}{\partial k}=\frac{t}{r}\left(\frac{t^{2} E}{t^{2}-r^{2}}-F\right)-\frac{r t^{2}}{R\left(t^{2}-r^{2}\right)} \sqrt{\frac{R^{2}-t^{2}}{R^{2}-r^{2}}},
$$

so that

$$
\frac{\partial[t \mathcal{K}(r, t)]}{\partial t}=-\frac{1}{t}\left(\frac{t^{2}}{t^{2}-r^{2}} E-F\right)-\frac{R}{\sqrt{R^{2}-t^{2}} \sqrt{R^{2}-r^{2}}} .
$$

To get (3.16), it remains to substitute (3.20) and (3.22) into the right-hand side of (3.8).

\section{Acknowledgements}

The research was supported by Russian Federal Targeted Programme "Scientific and Research-Educational Personnel of Innovative Russia" for 2009-2013, Project N 02.740.11.5024.

The author thanks Alexey Gil for the comments which improved calculations in the proof of Lemma $\mathbf{3 . 3}$. 


\section{References}

[1] I.S. Gradshtein and I.M. Ryzhik, Tables of Integrals, Sums, Series and Products. 5th Edition, Academic Press, Inc., 1994, 1204 pages.

[2] G. Iovane and M. A. Sumbatyan, Numerical treatment of hypersingular integral equations with application to crack mechanics, 2003, arXiv:math-ph/0307025v1.

[3] H. Rafeiro and S. Samko, On multidimensional analogue of Marchaud formula for fractional riesz-type derivatives in domains in $\mathbb{R}^{n}$. Fract. Calc. Appl. Anal., 8, No 4(2005), 393-401.

[4] N.A. Rostovtsev, An integral equation encountered in the problem of a rigid foundation bearing on nonhomogeneous soil. Prikl. Mat. i Mekh. 25 (1961).

[5] B.S. Rubin, One-dimensional representation, inversion and certain properties of Riesz potentials of radial functions. Mat. Zametki 34, No 1 (1983), 521-533.

[6] B.S. Rubin, Fractional integrals and weakly singular integral equations of the first kind in the $n$-dimensional ball. J. d'Analyse Math. 63 (1994), 55-102.

[7] B.S. Rubin, Fractional Integrals and Potentials. Pitman Monographs and Surveys in Pure and Applied Mathematics, 1996. Vol. 82, 409 pages.

[8] S.G. Samko, Hypersingular Integrals and their Applications. Taylor \& Francis, London-N. York, Series "Analytical Methods and Special Functions", Vol. 5, 2002. $358+$ xvii pages.

[9] S.G. Samko, A.A. Kilbas, and O.I. Marichev, Fractional Integrals and Derivatives. Theory and Applications. Gordon \& Breach. Sci. Publ., London-New-York, 1993, 1012 pages. (Russian Ed.: Fractional Integrals and Derivatives and Some of Their Applications. Nauka i Tekhnika, Minsk, 1987.)

[10] M. Sumbatyan and A. Scalia, Equations of Mathematical Diffraction Theory. CRC Press, Series "Differential and Integral Equations and Their Applications", 2004.

Universidade do Algarve

FCT, Campus de Gambelas

Faro 8005-139, PORTUGAL

e-mail:ssamko@ualg.pt

Received: July 10, 2010 